\title{
Effects of Autonomous Driving on the Vehicle Concept
}

\author{
Hermann Winner and Walther Wachenfeld
}

\subsection{Introduction}

Since Carl Benz invented the automobile in 1886, some very different vehicle concepts have been developed. Some can be regarded as the logical continued development and replacement of former concepts, such as the renunciation of the carriage design and the integration of the wheels and chassis under the (self supporting) body. The main factor that influences the vehicle concept is the purpose. This can particularly be seen when considering commercial vehicles. However, a wide range of passenger cars has also been developed, from convertibles and SUVs characterized by the owner's lifestyle to multi-purpose vehicles with sedans and hatchbacks as well as station wagons and (mini) vans. The type of use is even more important for small delivery vans, minibuses and what are known as light trucks on other markets. Under the hood, there has been a move toward transversely installed front engines with front-wheel drive over the past decades, after the dominance of longitudinally installed engines with rear-wheel drive. Furthermore, there is currently a trend towards electrification of the power train, deemed more sustainable due to $\mathrm{CO}_{2}$ specifications, less noise emission and other advantages. Despite all these changes, it is not expected that the existing drive concepts will "die out", since the divergence in the optimization aims of the different market segments will continue to support this diversification of concepts. For example, a rural area in the USA has completely different drive requirements to a big city in China.

H. Winner $(\square) \cdot$ W. Wachenfeld

Technische Universität Darmstadt, Institute of Automotive Engineering-FZD,

64287 Darmstadt, Germany

e-mail: winner@fzd.tu-darmstadt.de

W. Wachenfeld

e-mail: wachenfeld@fzd.tu-darmstadt.de 
The diversity of vehicle models available on the market is accompanied by the concept diversity of "show cars" presented at motor shows. Assistance systems and partial automation are only concept changing in very few cases for familiar vehicles. The visible trend over the past years of poorer outward visibility in vehicles, justified by styling requirements and/or requirements relating to the body rigidity does, however, support the use of compensating systems such as ultrasound parking aids, rear-view cameras or the surround-view display. However, driver assistance systems generally do not change the concept, as only a few are included as standard equipment for a vehicle model series. As a result, the vehicle manufacturer is required to design the vehicle so that vehicles without this equipment can also be safely driven by the driver. As the willingness and ability of the driver to take over are also prerequisites for partially automated driving [1], major changes compared with conventional vehicles cannot be expected. Only concepts that benefit a takeover, such as new human-machine interfaces for assigning a partially automated function may find their way in [2]. Not only former concepts, but also current drive-by-wire concepts with alternative control elements (see [3]) were restricted to replacing the function of the steering wheel and pedals without providing a design for the automation of the higher vehicle guidance levels that are indispensable for automated driving.

Although it is not yet possible to determine a trend toward another concept due to higher levels of automation, this may change with a considerable change in vehicle guidance. This results in the following question: If the opportunity of autonomous driving unsettles the current world of concepts, is this due to a significant shift in market shares or due to new concepts?

Before addressing this question in detail, the key conceptual features must be defined in order to create a level plane that applies to the overall vehicle and so that the even more diverse world of detailed solutions can be faded out. Prior to this, the requirements that will dominate concept selection in the future should be identified.

Figure 13.1 shows a selection of areas from which the requirements result. Many of the requirements are linked physically_ - often with conflicts — and can only be combined after a process of weighing up and prioritizing, for example streamlined aerodynamics versus distinctive styling components.

A distinction is made between the following domains

- Vehicle body

- Drive

- Chassis

- Interior

- Human-Machine Interface (HMI)

for this discussion of the top concept level. As major vehicle component clusters, these are always linked to the occupants and the environment, especially the road environment. The interaction between these areas can be represented abstractly as transfer of mass, energy and signals (see Fig. 13.2). Since vehicle automation radically changes this 


\section{Areas with requirements to consider}

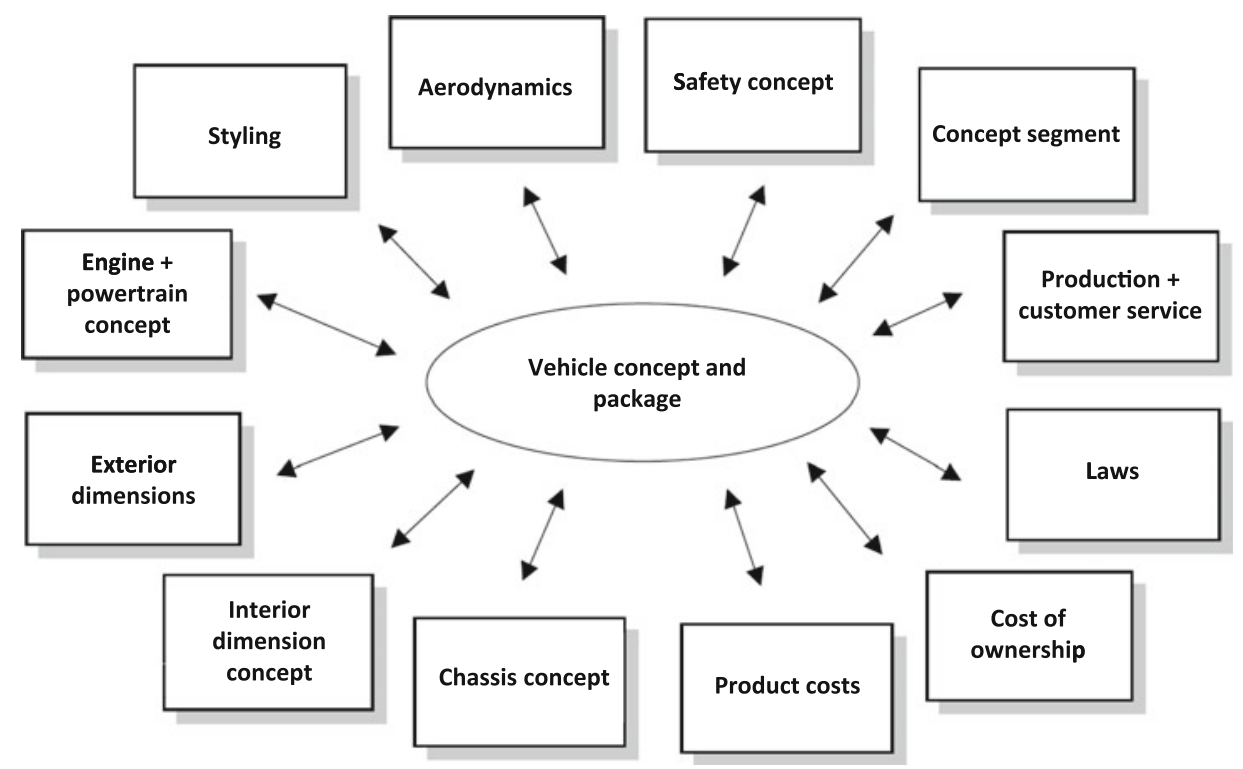

Fig. 13.1 Requirement areas for a concept decision [4]. Image rights: copyright by the Author
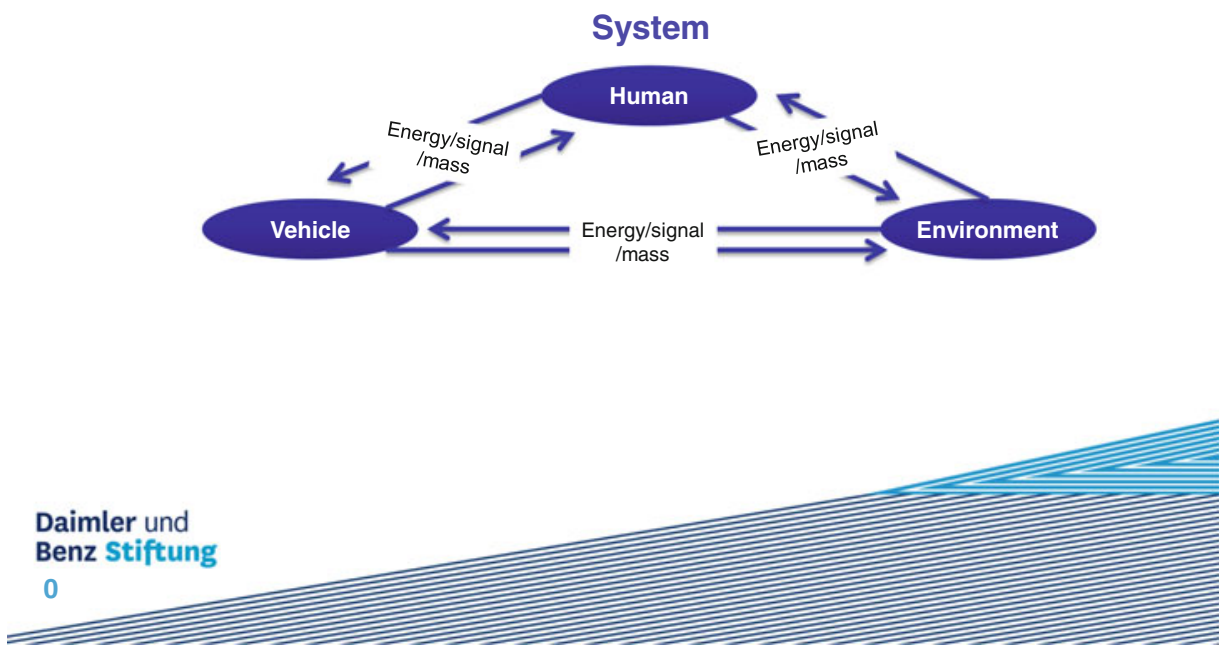

Fig. 13.2 System analysis: driver-vehicle environment. Image rights: copyright by the Author 
transfer, the modules are also affected. One example of the effect of automation is the exchange of signals between the environment and humans. The occupant no longer has to pursue the task of driving and therefore no longer has to exchange signals with the surroundings in order to reach the destination. This approach based on the transfer of mass, energy and signals makes the further structuring of the analysis possible, taking interfaces into account.

These concept-defining areas and their interfaces for the use cases selected in Chap. 2 as well as the application scenarios possible as a result are examined in the following.

\subsection{Interstate Pilot Using Driver for Extended Availability}

Although the two use cases "Interstate Pilot" and "Full Automation" have very different performance capabilities when it comes to autonomous driving, there are no differences when it comes to considering vehicle concepts, since both use cases are restricted to having a driver available for extended availability. For the "Full Automation" use case, autonomous driving can be used more extensively and a takeover is requested less often. However, in both cases it must be ensured that vehicle guidance is possible without restriction by a person with driver's license in their function as a driver for extended availability. Therefore, for both cases, the vehicle guidance is to be supported as a "mixed mode"-autonomous or manual.

\subsubsection{Effects on the Body Design}

Compared with the expected comparison concepts, which do not provide autonomous driving capability, no concept-defining change is perceptible for the planned continued manual vehicle guidance. For, as long as the extension and availability use-case concepts (see Chap. 2) are based on control by the driver, the degree of freedom when it comes to the body design is restricted. The external dimensions of the vehicle, as well as the positioning and size of the windows, must still be tailored to the driver, meaning that designs similar to those today are to be expected.

Sensors are required for virtually every viewing angle for autonomous driving, which will surely result in complex package solutions if discreet visibility is aimed for as to date. If a standard is actually to be set given for the capability to drive autonomously, this would be beneficial for the developers as they would be able to occupy outstanding sensor positions in the true sense of the word (see Fig. 13.3 as an example). Otherwise, no major deviation between the vehicle concept and comparison vehicles is expected, since these use cases "only" add a new feature to the existing concepts without affecting the basic principle of the vehicle or the application area. 


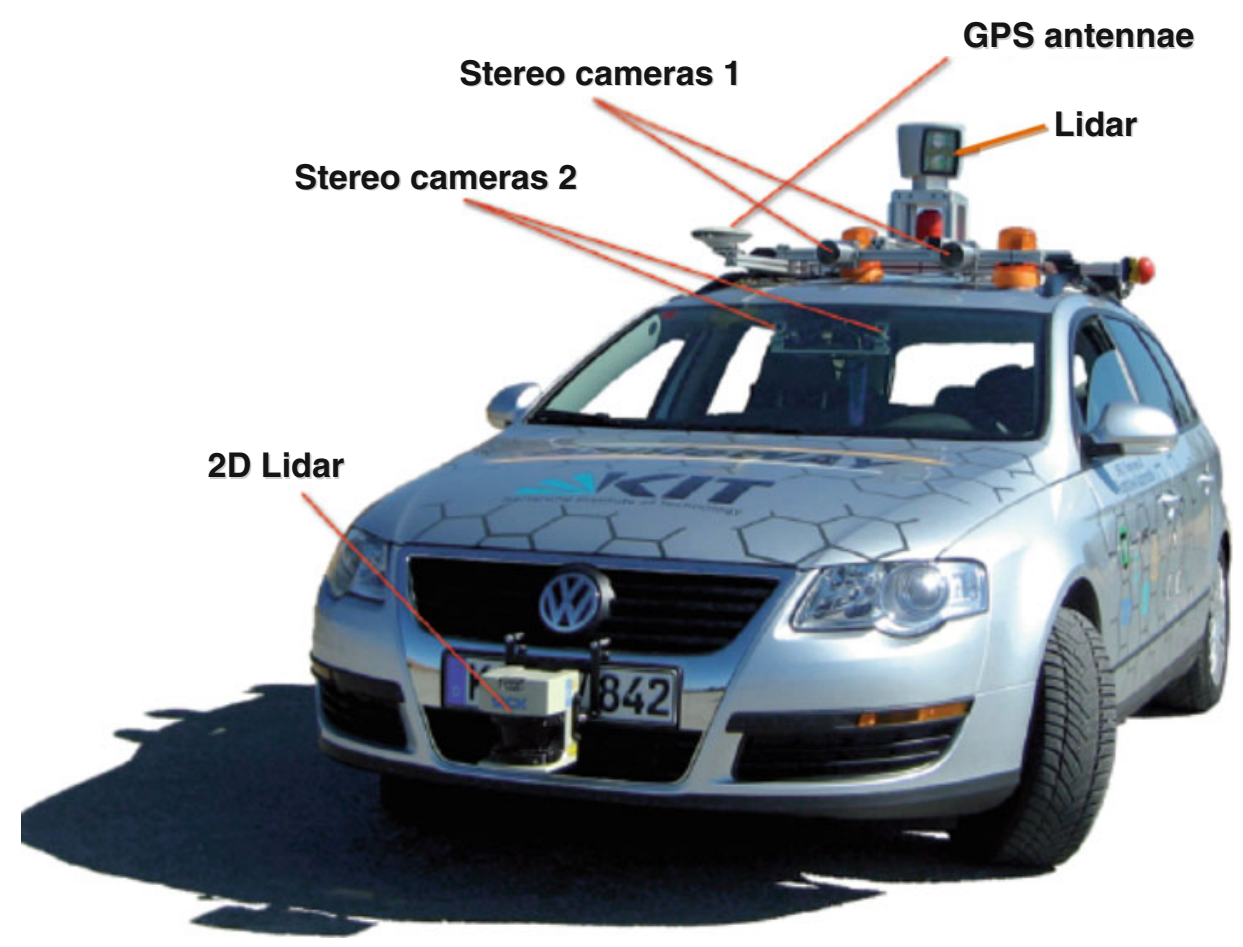

Fig. 13.3 Team anyway test vehicle, environment sensors [5]. Image rights: Springer

\subsubsection{Effects on the Drive Concept}

There is no reason to evaluate the drive concept differently for the potentially remaining manual journey segments than for similar vehicles that are not autonomous. However, autonomous driving simplifies incorporation into the traffic telematics compared with manual driving, making more efficient driving possible. The driving characteristics can be controlled depending on time and energy requirements. In principle, these options are already available on vehicles with partial or highly automated driving functions, meaning that the next step towards autonomous driving results in barely any relevant changes to the drive concept. It is more the new networking options which benefit the drive concepts with lower overall availability, such as battery-electric drives.

\subsubsection{Effects on the Chassis Design}

While manual driving needs a chassis with the standard requirements, long phases of autonomous driving enable the occupant(s) to be decoupled from the driving process. Automation needs neither tactile sensitivity for driving dynamics nor the transverse and 
longitudinal force action which impacts the occupants with vehicle acceleration. It can revert to the vehicle motion sensors that have long been in use for electronic stability control in order to regulate the trajectory so as to make the latter dynamic and comfortable.

A tilted chassis as available for rail transportation for some time could at least compensate for the standard moderate lateral accelerations of $1-2 \mathrm{~m} / \mathrm{s}^{2}$. This would require an overall tilting angle of approximately $6-12^{\circ}$. This overall tilting angle can be represented by the tilting angle of the road surface, the vehicle configuration roll angle and the seat angle, see Fig. 13.4. It seems possible that lateral force compensation can be achieved using the chassis alone, as the 2014 market launch in the S-Class Coupé model of Mercedes-Benz initially shows. By contrast, the deflection and rebound required for longitudinal force compensation is more than double the current spring travel. To achieve a compensation in the specified range here too, this would only be possible by implementing (separately or additional) seat tilting.

As fascinating as the idea of a journey free from longitudinal and lateral forces is, especially for rest periods, it is equally problematic with respect to the desired visual connection between humans and their environment, since the visual impression no longer matches the kinesthetic and vestibular impression. However, even with visual decoupling, the rotational acceleration which is sensed in the vestibular organ can cause occupants to feel unwell. This is because, unlike translational acceleration, this cannot be compensated for if the passenger cell cannot be turned against the vehicle's direction of travel. In addition, it is not possible to adjust the tilt angle without rotational velocity and accelerations, which significantly reduce the tilt dynamics. Thus, with a perception limit for the rotational acceleration (rolling) of $4 \% \mathrm{~s}^{2}$ as determined in experiments [6, 7], the target angle for acceleration compensation is not achieved for $2.5 \mathrm{~s}$. With a very anticipatory autonomous driving style where there are no external interferences, such as other vehicles which have to be reacted to with high changes in acceleration, this can just still be considered in trajectory planning. The perception limit used is the result of an experiment and therefore dependent on the underlying test setup. Other experiments give different values depending on the corresponding test setup, the rotation axis and the test person $\left(0.3-6^{\circ} / \mathrm{s}^{2}\right)$. A compilation of different studies into this topic can be found in [7-9].

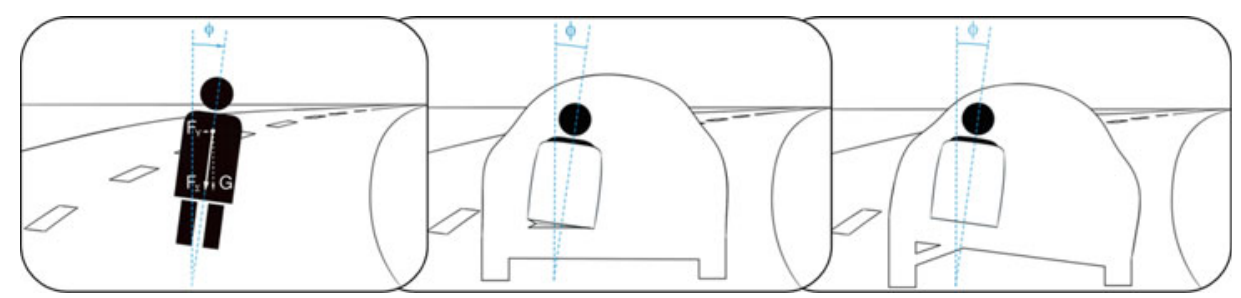

Fig. 13.4 Tilting concept when cornering: left addition of forces impacting on occupants; center seat angle; right angle by means of chassis. Image rights: copyright by the Author 
Compensating for the disturbance from vertical forces seems to be simpler. In conjunction with the front surroundings sensors, the feeling of a sedan chair should be possible. For reasons of safety this decoupling should be deactivated in order to give priority to the driving dynamics. This sedan chair could be based on electronically controlled air suspension concepts with adjustable dampers or fully active electromechanical spring-damper units. Relevant counter measures generally have to be taken in order to avoid low-frequency pitching movements which cause "seasickness".

\subsubsection{Effects on the Vehicle Interior and Human-Machine Interaction}

The necessity of an available driver means that a driver's workplace must always be provided. For this reason, the arrangement of the vehicle interior will be characterized by the need for a seat with a dashboard and operating elements. This means that no major concept changes are to be expected within the framework of the use cases considered (Interstate Pilot and Full Automation). As an alternative to the currently familiar human vehicle control using a steering wheel and pedals, an alternative control concept may be implemented, drawing on partially automated basic functionalities. This could also be linked with other space-saving control elements. Otherwise, several concept vehicle examples include the option of moving the control elements, particularly the steering wheel, to a less disturbing position, meaning that the space in front of the driver is available for the "alternative program" during autonomous driving. Some kind of lock for the operating controls may also be required so that they are not accessed accidentally, causing an unintentional takeover.

As well as using the driver's workstation as a mobile office for work or a media platform for entertainment, autonomous driving also allows the travel time to be used for relaxation or communicating with fellow passengers. Accordingly, for vehicles with this use case, the seating concept is expected to accommodate the respective requirements, although a lot of it is already implemented in current vehicles, even if not for the driver's seat.

\subsection{Autonomous Valet Parking}

Autonomous valet parking can help to solve the parking space problem for private car owners in residential areas or at workplaces and, for car-sharing users, can bridge the gap between the car-sharing parking position and the desired point of entry or exit (Chap. 18). Apart from this feature, the vehicle is a completely "normal" vehicle, which provides the existing capabilities for assisted, partially or highly automated driving.

When there are no occupants or objects in a vehicle, it is possible to use the free space in the vehicle with a variable body concept. Concepts with this function already exist 
today, enabling the dimensions of a vehicle to be reduced during parking. However, the authors are not aware of any use in series production, probably because the requirements for high passive safety clash with the requirements for a variable body. Although autonomous valet parking is advantageous for this type of space-saving due to lower parking rates, at the same time it reduces pressure on making the most of the space available, since the route to a large parking space is now tackled without cost or time-pressure. It is difficult to predict whether the fuel consumption for the route to the parking space ends up being more than for searching for a parking space.

Otherwise, autonomous valet parking does not result in any other fundamental degrees of freedom for the vehicle concept.

\subsection{Vehicle-on-Demand}

The vehicle-on-demand use case refers to a completely driver-less vehicle which does not have a driver's workplace and cannot fall back on the driver's capabilities. This results in new degrees of freedom for the design of these vehicles. The following describes these for this use case. Since this concept favors use over ownership, it can be assumed that in most cases the user neither owns nor has ownership responsibility for the vehicle. Instead, service providers make these vehicles available for mobility services (Chap. 18).

\subsubsection{Effects on the Body Design}

For the use case with driving speeds of up to $120 \mathrm{~km} / \mathrm{h}$, which covers almost all areas of use, the vehicle body must provide the occupants with weather and impact protection in line with that of current vehicles. This is associated with considerable design restrictions. A closed passenger compartment, possibly with a convertible roof, is required for weather-protection reasons. A safe shell with restraints must also be planned for the passengers in order to guarantee the same passive safety as in current vehicles, with seat belts and airbags. This requirement will exist for as long as it is not possible to ensure accident-free operation in road traffic, which is currently not conceivable, at least in mixed traffic with driver-only vehicles. The vehicle shape may correspond to that of current vehicles depending on the purpose and target group, with a large range of variants possible. One-box cars provide the best space economy. Like a cuboid, they are not lowered at the front or rear of the vehicle. Of course, designers will also make an effort to present the selected shape to appeal to the customers. However, with the wide range of possible uses for a vehicle-on-demand concept, the styling target is still largely unclear, especially to what extent individual emotions will be catered to. A look at the history of road vehicles shows that there are examples for almost every body design that are regarded as aesthetically successful or unsuccessful. As a result, it is not possible to predict a trend from the shape alone. However, all vehicles that include speeds higher than 
the $120 \mathrm{~km} / \mathrm{h}$ specified here will have aerodynamic state-of-the art shapes, meaning that they will have curves and aerodynamic tapers and air flow break-away edges [10].

As well as its protective function, the shell around the passengers is also responsible for connecting the passengers with the environment. In principle, full decoupling is possible as there is no need to guarantee that the driver can see. This enables private use of the cabin for entertainment and relaxation, virtually free from visual and acoustic disturbances. However, the influence of driving dynamics can only be insulated to a certain extent, as described in Sects. 13.2.3 and 13.4.3. There will always be the challenge of guaranteeing that the impressions of the different senses correspond, as otherwise this results in discomfort or even sickness, which is unlikely to result in insulation concepts of this type being accepted. Figure 13.5 shows what a complete decoupling during the journey would look like on public roads.

The range of alternative activities made possible by this decoupling is large and ranges from reading in a relaxing "environment" (right-hand vehicle) to exciting entertainment on racing tracks (left-hand vehicle). The challenge of guaranteeing that the impressions of the different senses correspond also applies to the artificial representation of the environment. The option of selecting the degree of decoupling using manually operated covers (blinds) in the simple case to high-tech solutions such as electro-chromic windows definitely makes it easier to reach a compromise. A virtual connection with the environment in the form of live camera images of the environment which can be shown (on screens, as a projection) is also conceivable, but requires a very high level of passenger trust in the

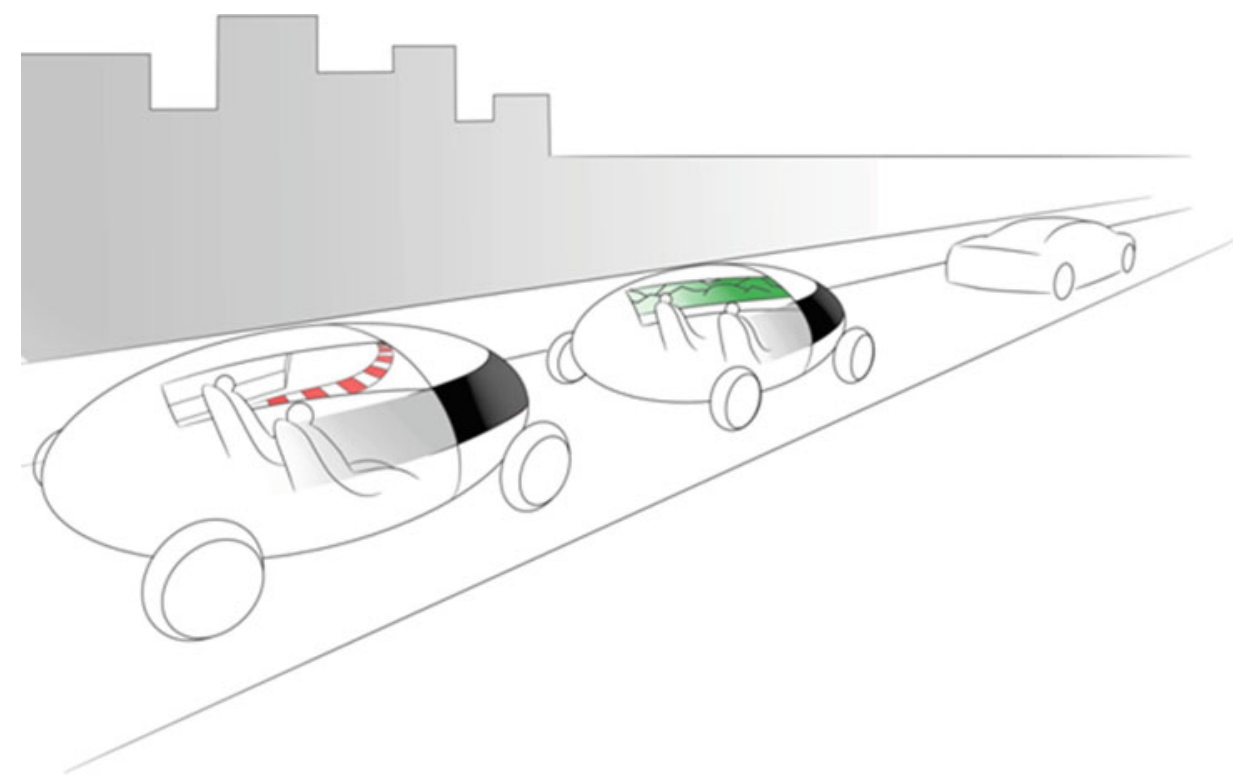

Fig. 13.5 Decoupling the passengers from the real road traffic. Image rights: copyright by the Author 
reliability of the technology. Traveling in an autonomous vehicle should not be associated with the fear of being exposed to an insulation cell without contact with the environment, even temporarily. A compromise between the virtual and real world is the provision of information about the environment in the form of an "augmented reality", in which information about landmarks is shown as they are seen, for example.

\subsubsection{Effects on the Drive Concept}

It is not possible to derive any restrictions for the drive from the use case alone. Both classic combustion engines and electric drives as well as mixed forms (hybrids) are fundamentally suitable. Their advantages and disadvantages also apply for vehicle-on-demand. On the one hand, there are the storage and charging advantages of chemical energy that allow the vehicles powered by combustion engines to have a larger range, and on the other hand, the noise and pollutant emissions advantages of electric drives which are particularly valued in areas with a high population density. It must be ensured that there is enough energy on board for the intended trip for vehicle-on-demand. Therefore, a supply concept where a vehicle charges during time spent stationary is assumed. Suitable service points will offer this service both manually and automated, whether at conventional fuel filling stations or special electric charging stations. Inductive battery charging is particularly suitable here. Depending on how much the vehicle is used, the disadvantage of higher charging times for electric vehicles has varying impacts: If the stationary times are still higher than the traveling times, the charging times are generally part of the non-use time and therefore do not represent a high economic strain. This is different when the vehicles are intended for use almost around the clock. When this is the objective, the time required for energy provision is of considerable consequence, since charging the batteries results in a significantly larger drop in use than refueling vehicles powered by combustion engines.

(Hydrogen) fuel cell drives will be easier to implement with vehicle-on-demand concepts since a service provider-possibly even in association with other providers - can integrate the tank infrastructure into the process better than standard vehicle users. This means that parking spaces (= disposition points) and hydrogen filling stations can be coordinated, both with respect to the location and the process. The routes can also be designed based on the connection between the hydrogen filling stations from the start if one filling is not sufficient for the mobility service. Additionally, unavoidable evaporation losses that occur with hydrogen pressure tanks when stationary can be minimized to a large extent since the time spent stationary should be very low for vehicle-on-demand with economical operation. Despite the advantages of the vehicle-on-demand concept for (hydrogen) fuel cell technology, both channels, i.e. vehicle-on-demand and hydrogen drives need to achieve access to the market independently of one another. (Forced) coupling of the technologies for the market launch only reduces the prospects for both, 
since it results in only a fragment of the use options and therefore a limited market volume which is too small to justify the investment for both technologies.

\subsubsection{Effects on the Chassis}

Force transfer for vehicle-on-demand will continue to be the responsibility of the tires, since neither the advantages nor the disadvantages of alternative concepts (e.g. air cushions/propellers, magnetic levitation drive, chains) are significantly affected by vehicle automation. The driving dynamics requirements are also only going to change minimally, and the requirement of a particularly sporty chassis is unlikely to be included in the requirements specification for a vehicle-on-demand. The deceleration and evasion capability (i.e. acceleration of up to $\approx 10 \mathrm{~m} / \mathrm{s}^{2}$ ) will barely be lower than on current vehicles, even if a considerably more comfortable vehicle guidance is to be expected. However, with vehicle-on-demand, it is possible to plan the maneuver predictively in advance and thereby make the most of the opportunities provided by the existing driving dynamics, which could result in new opportunities for the chassis design.

As well as the two aspects discussed in the following sections, wheel layout and steering concepts, the same considerations regarding decoupling from the driving dynamics as described in Sect. 13.2.3 apply. This is particularly true for vehicle-on-demand vehicles with the purpose of transporting persons on longer journeys. Although decoupling the human driver from the driving dynamics and the environment requires a huge amount of technical effort, it would enable better correspondence of the different sense impressions than if only one part of the impressions were to be decoupled. A corresponding decoupling would also be useful for transporting sensitive goods.

\subsubsection{Possible Wheel Concepts for Vehicle-on-Demand}

If we consider the stabilization task for a human driver, vehicle-on-demand makes it possible to select a wheel concept without taking currently applicable requirements into account. This degree of freedom motivates existing concepts with fewer than four wheels to be reassessed for use as vehicle-on-demand.

As proven by motorbikes and three-wheelers, vehicles with fewer than four wheels are also suitable for transportation. In principle, one wheel alone is sufficient for movement, as unicyclists impressively demonstrate. Regulating the position of the center of gravity is the most important role here. A mechatronic system could take on the task of stabilization, enabling just about anyone to ride and control a one-wheeled vehicle. Two methods can be used here: Torque generation on the wheel and displacement of movable masses.

The first of these concepts is used on the two-wheeled SEGWAY ${ }^{\circledR}$ personal transporters [11]. "Weight shifting" via feet is sensed and the passenger angle is adjusted to suit the acceleration so that the resulting vector of gravity and inertial force always passes through the line connecting the wheel contact points. The same method is used for maneuvering with two wheels one in front of the other. A steering movement in the 
opposite direction to the direction of the corner results in a rolling moment which generates an angle which guides the resulting vector of centrifugal force and gravity through the line connecting the two wheel contact points. In principle, a ball drive could carry out this stabilization for both directions. However, it is still highly questionable whether implementation of this kind of stabilization is useful in the context of autonomous driving. The user is always strongly incorporated with the weight support. Furthermore, these concepts are restricted to low speeds since vertical unevenness such as curb levels or potholes overburden both the control dynamics and the actuator capacity and therefore cannot prevent crashes. This consideration also applies to single-axle two-wheelers such as the SEGWAY PT mentioned above. These vertical obstacles are not a problem for motorbikes in transverse movement, even if phenomena such as kick-back reveal the sensitivity of vehicle stabilization of two-wheelers.

In the same way as tightrope walkers use their arms to balance, in the second concept, a mass is displaced in such a way that the center of gravity is moved to suit the longitudinal or lateral forces without any help from the occupants. To stabilize this displacement, torque is briefly applied to the wheels. The SEGWAY P.U.M.A. (Personal Urban Mobility and Accessibility) concepts [12] and the implementation by General Motors as an EN-V (Electric Networked-Vehicle) [13] are used in connection with autonomous driving. The good maneuverability (turning on the spot) and lower space requirement mean that this vehicle is well suited to urban use, and this is, of course, not only restricted to autonomous driving. The disadvantages are the permanent stabilizing effort which "consumes" a larger share of the energy on board and the sensitivity to vertical levels specified above, although the design results in this being less than expected since the movable mass can reduce the drive torque required for correction here. According to statements to date, the maximum speed is planned to be 25 miles an hour, or $40 \mathrm{~km} / \mathrm{h}$. This concept is hardly suitable for use up to $120 \mathrm{~km} / \mathrm{h}$.

Movable masses can also be used for the classic single-track two-wheeled layout, although it is more difficult to satisfy the space requirement for the displacement option in the transverse direction than in the longitudinal direction for a single-axle two-wheeled vehicle. For this reason, a steering actuator that can carry out balancing seems more promising for autonomous motorbike guidance. However, it remains questionable whether a concept like this can comply with the mobility requirements for a vehicle-on-demand. Full enclosure is cumbersome and significantly increases side wind sensitivity. However, it should not be ruled out as, in addition to weather protection, an enclosed frame also considerably improves safety, as shown by the BMW C1 scooter produced from 2000 to 2003 [14]. Equipped in this way, a single-track two-wheeled vehicle-on-demand is conceivable, possibly including stabilizers for very low speeds and when stationary. However, whether the special mobility of single-track vehicles, such as passing queues of traffic, can be implemented with autonomous driving is another question.

The two approaches have an opportunity in common: They can both provide force-free driving in one direction. On the single-axle two-wheeled vehicle, the longitudinal force on the occupants can be fully compensated for, and on the single-track two-wheeled vehicle 
the same applies for the transverse force. It is an open question whether a passenger experiencing the trip as a passive occupant finds this to be pleasant.

With three or more wheels, a vehicle is stable without control as long as the force vector resulting from inertial force and gravity does not leave the area between the connection lines surrounding the wheel contact points. Depending on the height of the center of gravity $h_{\mathrm{s}}$ and the driving dynamics limits to be taken into account due to the maximum friction coefficient $\mu_{\max }$, the area of the resulting vector with the road surface is a circle with radius $r_{\text {res }}=h_{\mathrm{S}} \cdot a_{\max } / g=h_{\mathrm{S}} \cdot \mu_{\max }$ around the central point, see Fig. 13.6. The central point is the result of projecting the center of gravity in the $x-y$ area of the road surface. The driving dynamics limits result from the (absolute value of) maximum longitudinal and transverse acceleration in relation to gravity acceleration $g$ (assumed to be the same for both directions here $a_{\max } / g$ ). If the outer connection lines of the wheel contact points intersect this circle, there is a danger of rolling over. If they are outside of the circle, it is still possible that the vehicle may roll over in unfavorable conditions, as proven by the well-known example of the first Mercedes-Benz A-Class (further details are available in [15]).

The minimum footprint area required to resist rolling over on a three-wheeled vehicle, determined by the wheel contact points, is $A_{\mathrm{FP}, 3}=3 \sqrt{3} r_{\text {res }}^{2}$ and thereby approximately $30 \%$ larger than the quadratic area. More problematic is the $50 \%$ larger minimum width of a three-wheeled vehicle, which unlike in Fig. 13.6 is based on two corner points in the longitudinal direction (otherwise $73 \%$ larger width in the position shown). For this reason, a three-wheeled vehicle with purely static support will be unlikely to be used for automated driving.

\section{Footprint for tilting stability}

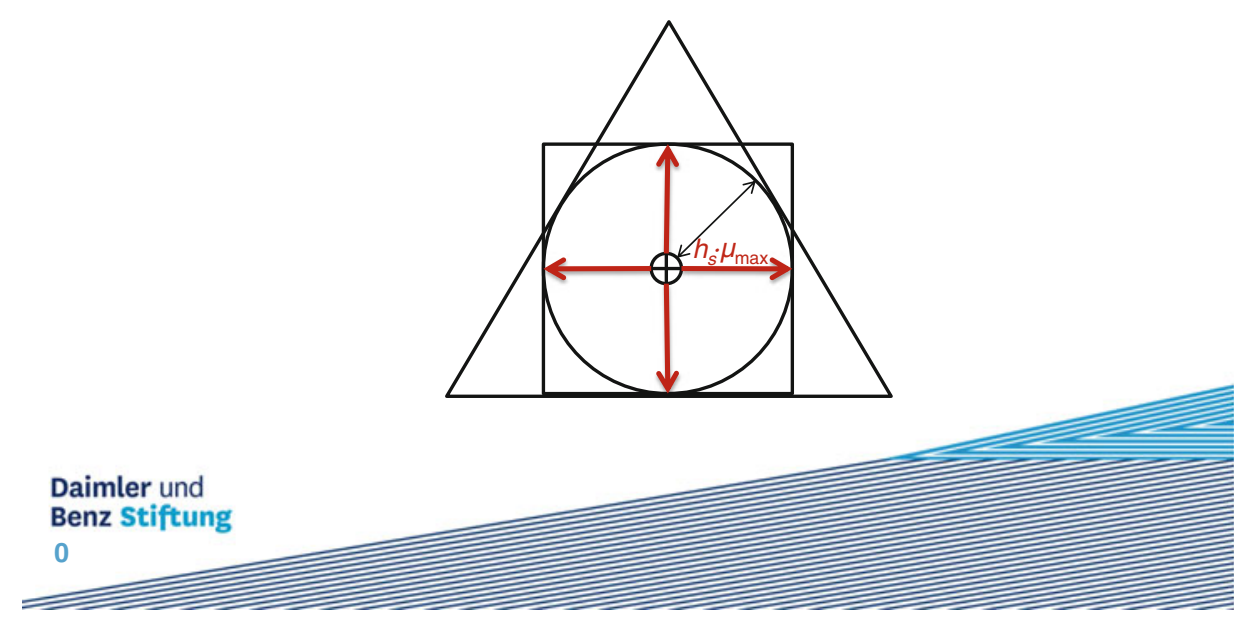

Fig. 13.6 Footprint area required for resistance to rolling over. Image rights: copyright by the Author 
A hybrid chassis solution with a footprint that is too small for static support makes it possible to displace the center of gravity so that the circle drawn in Fig. 13.6 is displaced in relation to the chassis, e.g. moves to the left in a left-hand bend. The displacement can be represented using a translational movement or a rotational movement. The Piaggio MP3 three-wheeled scooter design is an example of the latter [16] with two front wheels and parallelogram kinematics enabling an angle for the complete scooter structure. As a result, this vehicle has a restricted static tilting stability, however it does not require permanent control for constant conditions, e.g. for driving on straight roads. This type of concept would definitely be an option for vehicle-on-demand in conjunction with the enclosure mentioned above and a protective frame, if the transport requirement is to transport one, or maybe even two people.

Similarly, a third wheel can also complement a single-axle concept such as the EN-V mentioned already. Here, a wheel affixed in front of or behind the main axle is responsible for static support. However, the retention of rotation while stationary requires that the additional wheel can be steered. Four-wheel designs with a footprint that is too small to resist rolling over are conceivable. An example of this is the Nissan Land Glider study [17], a vehicle with a width of $1.10 \mathrm{~m}$ which can assume a lateral angle of up to $17^{\circ}$. Whether this still relatively large width compared to a scooter is accompanied by the advantages for a vehicle-on-demand compared to a standard vehicle of normal width without tilt technology is disputable. The ability to compensate for the transverse force on the occupants using tilt technology could be a plus point in its own right. As well as tilting systems, adaptive chassis with a variable track width or a variable wheelbase and variable center of gravity height could fundamentally also result in resistance to rolling over as required [18].

\subsubsection{Steering Concepts}

Currently, axle pivot steering on the front axle is dominant, and there is little reason to not use this for vehicle-on-demand too. However, the status of alternative steering concepts could be enhanced by autonomous driving. These include steering by forces like a skid steer, which can range from different forces to different force directions on one axle, e.g. a negative driving force on the left and a positive driving force on the right for turning to the left. This concept is the method of choice for the single-axle two-wheeled vehicle. The steering by forces results in considerable restrictions for three or four-wheeled vehicles if there is not at least one additional rotational degree of freedom for the wheels to support steering control.

Alternatively, multi-axle steering is possible, often referred to as four-wheel or all-wheel steering. Although this is known of in vehicle technology, there are only very few vehicles available with it. Use is restricted due to the compromise design of current all-wheel steering vehicles which vehicle developers have to carry out. The driver cannot access the rear wheel steering. It is coupled (electronically) to the front wheel steering. 
In a trade-off, it currently supports the vehicle agility at lower speeds $(\lesssim 100 \mathrm{~km} / \mathrm{h}$ ) with inverse steering angles and the stability at higher speeds $(\gtrsim 100 \mathrm{~km} / \mathrm{h})$ with concordant steering angles. Steering corrections are also carried out in the critical limits of driving dynamics. Autonomous trajectory planning could use this degree of freedom independently of the speed and in accordance with the planned maneuver. This would make it possible to change lanes without yawing, i.e. without rotation around the vertical axis, allowing the repercussion on the passengers to be reduced. When parking, maneuvers are possible that are not possible with all-wheel steering as in a standard set-up, far less with standard front-axle steering.

An extreme solution would be all-wheel steering that controls each wheel individually. This would be able to position all the wheels in an optimum angle but which requires more complicated actuator systems. There is little advantage for the application as a vehicle on-demand. It is restricted to a displacement of driving dynamics limits of a few percent, principally relevant for racing and a reduction in cornering resistance due to restriction-free wheel control.

\subsubsection{Effects on the Vehicle Interior and Human-Machine Interface}

If the vehicle on-demand is used to transport passengers, the possibilities specified in Table 13.1 are available for the type, direction and positioning of passenger accommodation. There is fundamentally no reason not to continue with the current usual positioning of seats in front of and beside each other, facing forwards. If occupant protection allows it, it is possible to deviate from this, and the fully reclined position in particular should be offered for relaxation, although the direction remains open, e.g. as a row of seats in the transverse direction or as a reclining seat in the longitudinal direction.

As the occupants are not expected to take on any driving tasks, the interface is essentially restricted to entering the destination, occupant information and a safe-exit switch which causes the vehicle to stop at the next safe point. The first two could even be separated from the vehicle and could be implemented using a personal device similar to today's smartphones. Advanced interactions are to be expected for controlling the ambiance (e.g. closing the (electronic) blinds) and any entertainment programs.

Table 13.1 Generally possible accommodation of occupants - type, direction and position

\begin{tabular}{|c|c|c|c|c|}
\hline Type & Standing & Sitting & Lying & Variable \\
\hline Alignment & To the front & To the side & To the rear & Variable \\
\hline Position & In front of each other & Next to each other & On top of each other & Variable \\
\hline
\end{tabular}




\subsection{Use Case Overall View}

Autonomous driving varies less by vehicle concept than by the use of driving time. With the current statistical vehicle occupation of 1.5 persons [19], the majority of these persons cannot make use of the options available to passengers today, such as reading, working or sleeping, see Fig. 13.7. Autonomous driving makes this possible. Additionally, activities are possible which would currently result in impermissible distraction of the driver, e.g. from multimedia entertainment on a large screen and surround sound to what is referred to as 4D cinema.

There is a further change to the user group. Previously, a driver with driving authorization was always required. With vehicle-on-demand, this restriction no longer applies, meaning that new users who could currently only be passengers in the vehicle can use the autonomous vehicles. These could be people who are not permitted to drive due to physical disabilities (e.g. visual impairment) or persons who are not able to drive due to mental health issues or simply groups such as children and young people who are not permitted to drive due to their age. Accordingly, the vehicle and the operational concept must also be adapted for these users. First, it is necessary to define the requirements as to what authority the occupants have over what happens in the vehicle and on the driving.

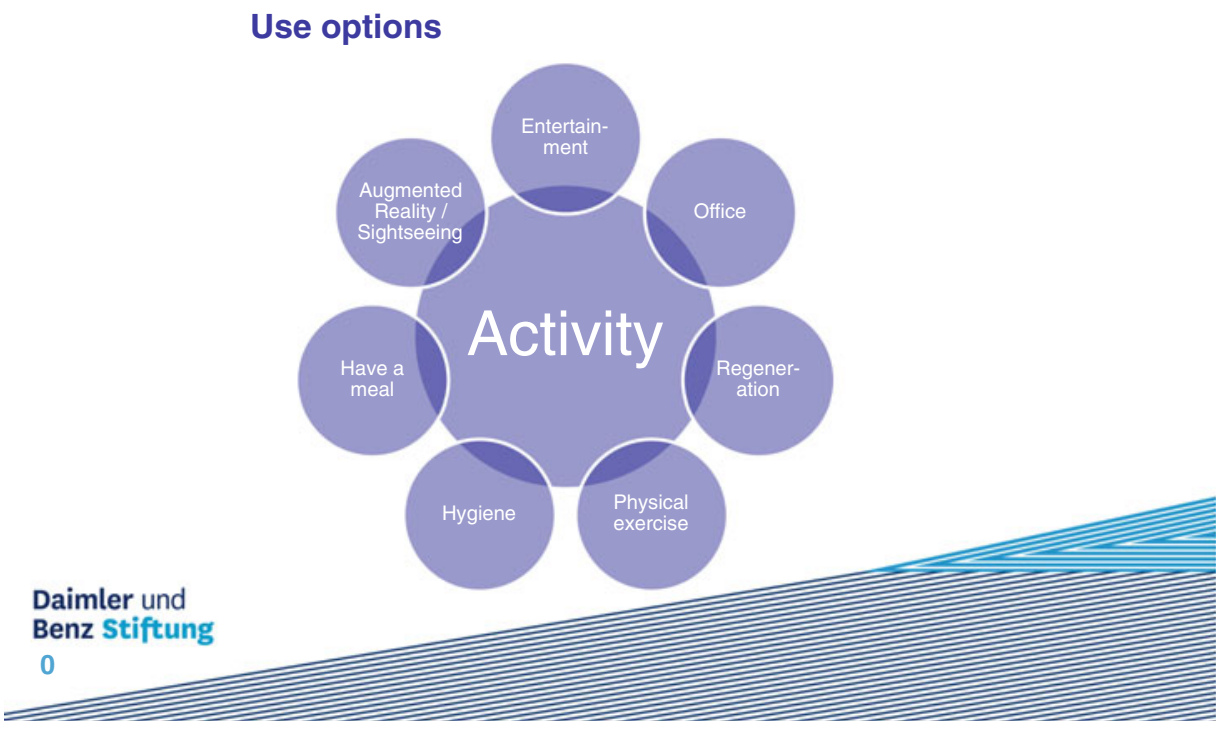

Fig. 13.7 Usage options for occupants during autonomous driving. Image rights: copyright by the Author 


\subsection{Changes that Go Beyond the Vehicle}

If the focus so far was on the individual vehicles, the system boundary is expanded in this section to include interaction with the environment and other road users. The interconnectivity of vehicles will be established as technology for all (new) vehicles early on, meaning that the introduction of autonomous driving does not result in anything fundamentally new. However, automation of vehicles also increases their performance capability, making new forms of use possible. The higher level of precision expected in autonomous vehicles means that electronically controlled maneuvers are possible which would scarcely be conceivable in this form in current traffic. These opportunities include convoy driving with very small distances between the vehicles or driving in particularly narrow driving lanes. The impact on the flow of traffic can be seen clearly in Fig. 13.8. The number of traffic elements that cross the depicted traffic area per time unit increases. If this is coupled with the corresponding pre-emptions, this can boost autonomous driving. Depending on the specifications associated with these pre-emptions, the vehicle concept may also change, and even the appearance may change with these specifications. The extent to which a regulation like this can affect the vehicle concept can be seen in the restriction of the overall vehicle length of trucks in the EU. As a result, all tractive vehicles in Europe are equipped with a sheer, space-saving vehicle front, while vehicles in North

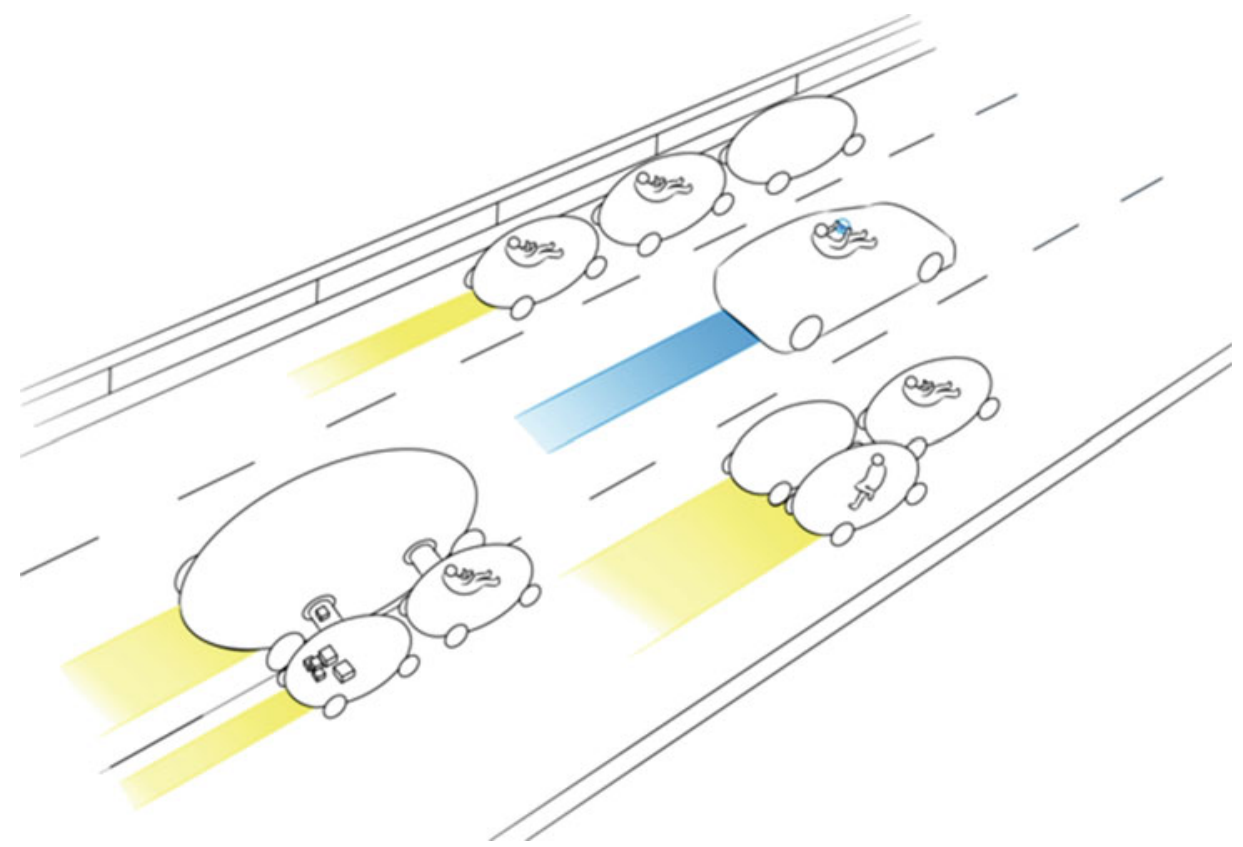

Fig. 13.8 Reduced distances between vehicles - made possible by high-precision autonomous driving. Image rights: copyright by the Author 
America that are not subject to this restriction have an aerodynamic hood extending forwards.

Mass transfer during the journey is conceivable, if still very unusual from today's perspective. This is already implemented in agriculture (e.g. unloading crops while reaping) and in aviation in the form of refueling while still in the air. This could save time and space when transporting goods or passengers. Accordingly, suitable transition areas would have to be included in the vehicle design. This principle is illustrated in Fig. 13.8. A long-distance vehicle similar to a truck transfers goods to the smaller "distributor vehicle" during the journey. Automation means that the journey is synchronized precisely and a bridge-like construction makes it possible to exchange goods. This means that completely new logistics and mobility concepts can be developed. However, without discussing the overall system, designing a vehicle does not make much sense, which is why this is not covered in more detail here.

\subsection{Follow-Up Concepts}

While the use cases examined throughout have the potential to help with the breakthrough of the technologies required for autonomous driving, a number of applications that alone would not be sufficient to help a technology to reach the required maturity would benefit from this. This would mean that roadworthy service robots would be an option, for example to clean roads, cycle lanes and pavements and move independently from one job site to the next using public roads. Other transport and utility vehicles that were formerly restricted to depots could now leave them, or, in other words: Depots are expanded to public grounds.

This eliminates the boundaries that were placed on autonomous systems to date, both with respect to scaling (from household robot to walking frame and cleaning robot, to post delivery vehicle and patient transportation) and relating to the private, public and commercial areas in which they are implemented. These concepts would have a big impact on the world of work and could reduce the number of people employed in the mobility sector in a wave of rationalization. This use case, following the vehicle-on-demand concept, has the potential to considerably reduce the number of professional driver jobs in particular. As well as the world of work, these vehicles could also revolutionize access to goods in particular, since it would be possible to automate the path from request for goods to the physical delivery (Chap. 16). Pilot projects with multi-copter "drones" are already sketching out this path for light goods. The unbeatable energy efficiency advantages of wheeled vehicles means that the demand for autonomous wheeled vehicles for the delivery of heavy goods is sure to come. 


\subsection{Summary}

In summary, the following three main points can be identified for concept changes after analyzing the four use cases:

With autonomous driving, no-one is bound to the task of driving for the duration of the trip. This results in freedom as to how to use this time. The different uses will have a considerable impact on the vehicle interior and the design of the human-machine interface.

Not having to take on the task of driving means that a change in the connection to the environment is possible. This applies both to the haptic channel for decoupling the effect of force from the road surface and driving dynamics on the passengers and to the visual decoupling. However, there are reservations here due to possible conflicts between visual and vestibular perception as can be observed in driving simulators today. This can only be solved by completely decoupling both worlds, i.e. when there is no visual impression from the outside and no force affecting the passengers. This would not only be very complicated to achieve for the chassis, but might also be alarming for passengers.

Since autonomous driving is always carried out by means of by-wire actuator control, a very high level of motion control accuracy can be assumed. On the one hand, this enables new steering concepts, possibly even connected with anti-roll over devices on vehicles with a footprint that is not sufficient for resistance to rolling over alone, especially if steering by humans is no longer planned. On the other hand, this precision enables connection to other autonomous vehicles with the same level of precision in order to better utilize the infrastructure or to enable concepts that still seem very unusual today, such as car-to-car mass transfer.

\subsection{Will Autonomous Driving Revolutionize Vehicle Concepts?}

Automation will not instigate a revolution in vehicle concepts. Although some concepts are less "disadvantaged" by automation, classic vehicle concepts such as four-wheel passenger cars are expected to continue to dominate in the world of automated road traffic. However, new opportunities arise for special niche use areas, only the appeal of these niches is driven more by the specific regulations and access restrictions than by the superiority of the concept for general individual motorized traffic.

As is already the case today, it will be the use that determines the concept for autonomous vehicles, and using the gained travel time will stimulate vehicle interior designs in particular.

Automated driving technology may well result in even more differentiation in the market than today:

On the one hand, there will be expensive, comfort-based high-tech vehicles resembling sedan chairs that serve as a moving living room, office or bedroom. 
On the other hand, low-cost utility vehicles will be used that have the equipment required for transport services but which resemble small city buses and are neither emotionally attractive nor especially comfortable.

The technology developed for the use cases specified above will result in lots of spin-off applications that may significantly change the service sector.

Open Access This chapter is distributed under the terms of the Creative Commons Attribution 4.0 International License (http://creativecommons.org/licenses/by/4.0/), which permits use, duplication, adaptation, distribution and reproduction in any medium or format, as long as you give appropriate credit to the original author(s) and the source, a link is provided to the Creative Commons license and any changes made are indicated.

The images or other third party material in this chapter are included in the work's Creative Commons license, unless indicated otherwise in the credit line; if such material is not included in the work's Creative Commons license and the respective action is not permitted by statutory regulation, users will need to obtain permission from the license holder to duplicate, adapt or reproduce the material.

\section{References}

1. Gasser, T. M.; Arzt, C.; Ayoubi, M.; Bartels, A.; Bürkle, L.; Eier, J.; Flemisch, F.; Häcker, D.; Hesse, T.; Huber, W.; Lotz, C.; Maurer, M.; Ruth-Schumacher, S.; Schwarz, J.; Vogt, W.: Rechtsfolgen zunehmender Fahrzeugautomatisierung [The legal consequences of increasing vehicle automation]. Joint final report by the "The legal consequences of increasing vehicle automation" BASt (Federal Highway Research Institute) project group, document part 1. Wirtschaftsverlag NW, Bergisch Gladbach, booklet F 83, 2012

2. Franz, B.: Entwicklung und Evaluation eines Interaktionskonzepts zur manöverbasierten Führung von Fahrzeugen [Development and evaluation of an interaction concept for the maneuver-based guidance of vehicles]. Dissertation, Institute of Ergonomics, Technische Universität Darmstadt, Darmstadt, 2014

3. Winner, H., Heuss, O.: X-by-Wire-Betätigungselemente - Überblick und Ausblick [X-by-Wire actuation elements - An overview and outlook]. In: Winner, H., Landau, K. (pub.): Darmstädter Kolloqium Mensch \& Fahrzeug - Cockpits für Straßenfahrzeuge der Zukunft [Darmstadt Colloquium Humans \& Vehicles - Cockpits for the road vehicles of the future], Technische Universität Darmstadt, Ergonomia-Verlag, Stuttgart, 2005

4. Futschik, H. D., Achleitner, A., Döllner, G., Burgers, C., Friedrich, J. K.-H., Mohrdieck, C. H., Schulze, H., Wöhr, M., Antony, P., Urstöger, M., Noreikat, K. E., Wagner, M., Berger, E., Gruber, M., Kiesgen, G.: Formen und neue Konzepte [Forms and new concepts]. In Braess, H.-H., Seiffert U. (pub.): Vieweg Handbuch Kraftfahrzeugtechnik [Vieweg Automotive Engineering Manual], 7th edition, Springer Vieweg, Wiesbaden, 2013, pages 119-219

5. Kammel, S.: Autonomes Fahren [Autonomous driving]. In: Winner, H., Hakuli, S., Wolf, G. (eds.) Handbuch Fahrerassistenzsysteme [Driver assistance systems manual], pp. 651-657. Vieweg+Teubner Verlag (2012)

6. Durth, W.: Ein Beitrag zur Erweiterung des Modells für Fahrer. [An article on expanding the model for drivers]. Fahrzeug und Straße in der Straßenplanung [Vehicles and streets in road planning] In: Straßenbau und Straßenverkehrstechnik [Road construction and road traffic technology], Bonn-Bad Godesberg, H 163, 1974 (1974)

7. McConnell, W.A.: Human Sensitivity to Motion as a Design Criterion for Highway Curves. Highway Research Board Bulletin (149) (1957) 
8. Fischer, M.: Motion-Cueing-Algorithmen für eine Realitätsnahe Bewegungssimulation [Motion-cueing algorithms for realistic motion simulation]. Deutsches Zentrum für Luft-und Raumfahrt in der Helmholz-Gemeinschaft [German Aerospace Center in the Helmholz Association], DLR (2009)

9. Betz, A.: Feasibility Analysis and Design of Wheeled Mobile Driving Simulators for Urban Traffic Simulation, Dissertation, Automotive Engineering department, Technische Universität Darmstadt, Darmstadt, 2014

10. Schütz, T.: Hucho - Aerodynamik des Automobils: Strömungsmechanik, Wärmetechnik, Fahrdynamik, Komfort [Hucho - Automotive aerodynamics: flow mechanics, heat engineering, driving dynamics, comfort], 6th edition, ATZ/MTZ-Fachbuch, Springer Vieweg, Wiesbaden, 2013

11. SEGWAY® Personal Transporter: http://www.segway.de/segway-pt/das-original, accessed on: $8 / 26 / 2014$

12. SEGWAY® Advanced Development, Project P.U.M.A: http://www.segway.com/puma, accessed on: 8/26/2014

13. GM EN-V Concept: A Vision for Future Urban Mobility: http://media.gm.com/autoshows/ Shanghai/2010/public/cn/en/env/news.detail.html/content/Pages/news/cn/en/2010/March/ env01.html, accessed on: 8/26/2014

14. BMW C1: http://de.wikipedia.org/wiki/BMW_C1, accessed on: 8/26/2014

15. Baumann, F.: Untersuchungen zur dynamischen Rollstabilität von Personenkraftwagen [A study of the dynamic roll stability of passenger vehicles], VDI progress report, series 12 no. 552, VDI-Verlag, Düsseldorf, 2003

16. Piaggio MP3: http://www.de.piaggio.com/piaggio/DE/de/news/MP3.html\#main, accessed on: $8 / 26 / 2014$

17. Schröder, C.: Tokyo Motor Show: Nissan präsentiert Stadtauto mit Neigetechnik [Nissan presents a city car with tilt technology], available under: http://www.springerprofessional.de/ tokyo-motor-show-nissan-praesentiert-stadtauto-mit-neigetechnik-10712/3946650.html, accessed on: 8/26/2014

18. Schweitzerhof, H., Betz, A., Winner, H.: Analysis of a situational adaptive chassis with respect to maneuverability and footprint. In: Proceedings of the ASME 2014 International Design Engineering Technical Conferences \& Computers and Information in Engineering Conference. IDETC/CIE 2014, Buffalo, New York, USA, August 17-20 (2014)

19. Institute for Applied Social Sciences (infas), Deutsches Zentrum für Luft- und Raumfahrt e. V. (German Aerospace Center): Mobilität in Deutschland 2008, Ergebnisbericht: Struktur, Aufkommen, Emissionen, Trends, [Mobility in Germany, 2008, Evaluation report: structure, emergence, emissions, trends] Bonn und Berlin, 2010 